\title{
Effect of the Charge Interactions on the
}

\section{Composition Behavior of Acrylamide/Acrylic}

\section{Acid Copolymerization in Aqueous Medium}

Eric J. Fischer, Danilo Cuccato ${ }^{1 *}$, Giuseppe Storti, Massimo Morbidelli

Institute for Chemical and Bioengineering, ETH Zurich, Vladimir-Prelog-Weg 1, 8093

Zurich, Switzerland

${ }^{1}$ Present Address: Department of Chemical Engineering, Imperial College London, South

Kensington Campus, London, SW7 2AZ, United Kingdom

*Corresponding Author: danilo.cuccato@chem.ethz.ch 
Acrylamide and sodium acrylate are copolymerized in aqueous solution to study the influence of monomer concentration and ionic strength onto the reactivity ratios using in-situ ${ }^{1} \mathrm{H}$ NMR. Increasing the monomer content leads to larger reactivity of the ionized monomer. At low monomer concentration, this effect was reproduced by adding $\mathrm{NaCl}$ to increase the ionic strength, indicating that the reaction kinetics is largely governed by charge interactions. On the contrary, this was not observed at higher monomer content, suggesting that non-electrostatic effects are mainly responsible of the monomer concentration dependence at these conditions. A comprehensive mathematical model was developed to predict copolymer composition as a function of monomer concentration and ionic strength. It is based on a previously-proposed rate law of propagation for ionized monomers, which has been expanded to cover any ionization degree of acrylic acid. The model is capable to reproduce composition data from different sources obtained in a wide range of reaction conditions.

\section{KEYWORDS}

acrylamide; acrylic acid; free-radical polymerization; reaction kinetics; electrostatic screening; reactivity ratios 


\section{Introduction}

Copolymers of acrylamide (Am) and acrylic acid (AA) are polyelectrolytes of great industrial relevance due to their wide range of applications. These water-soluble polymers exhibit peculiar properties in solution which make them suitable for several uses in separation processes and in the modification of the physical behavior of fluids. Am/AA copolymers are commonly employed as flocculating agents in waste water purification, to reduce drag in pipelines, in enhanced oil recovery by polymer flooding, and more generally as additives for thickening, binding, lubrication, crosslinking, etc. [1-4].

The many application properties of Am/AA copolymers and, more generally, of polyelectrolytes are primarily determined by their fundamental polymer features such as average length, composition, and microstructure of their polymer chains [5]. The possibility to tune these features during the polymer synthesis makes polyelectrolytes very versatile in the context of the industrial uses [6]. With this respect, comprehensive and reliable polymerization models represent invaluable tools towards the design of the reaction conditions suitable to control the polymer properties with enough accuracy. To this aim, a deep and reliable understanding of the kinetic behavior of such systems is a crucial requisite.

Another important factor affecting the properties and solution behavior of polyelectrolytes is represented by the electrostatic interactions originating from the charges within the polymer chains [7]. The nature and extent of such interactions are determined primarily by the electrostatic properties of the repeating units in the chains, namely the number, sign, and displacement of the monomer charges. In this context, the linear charge density of the polymer is a relevant parameter to correlate the monomer charge properties and the chain composition with the electrostatic features of polyelectrolytes [8,9].

Polyelectrolytes are often composed of ionizable monomers: in this case, the presence of ionizable moieties leads to the formation of charges on monomer and polymer depending on 
the degree of dissociation of the monomer units. For water-soluble ionizable species, the dissociation constants of the acid or basic groups determine the charge concentration of the monomer/polymer mixture in water. However, the degree of dissociation of such species can be adjusted by modifying the solution $\mathrm{pH}$ (i.e., upon addition of a strong base or acid to the system), especially when the ionizable monomers are weak acids or bases: this way, the solution $\mathrm{pH}$ becomes a relevant parameter to tune the magnitude of the electrostatic interactions of aqueous polyelectrolyte systems. Another factor influencing the extent of the charge interactions in polyelectrolytes is the ionic strength of the solution: namely, the addition of an electrolyte (e.g., $\mathrm{NaCl}$ ) to the system can induce a screening of the repulsion forces between the charges based on its concentration, according to the so-called phenomenon of electrostatic screening [10].

The electrostatic interactions arising from the presence of charges in polyelectrolyte systems can have an impact not only on the polymer properties but also on the reaction kinetics during the free-radical polymerization of ionized and ionizable monomers. For instance, the repulsion between equally-charged side-chain moieties of an ionized monomer molecule approaching the ionized terminal unit of a radical polymer chain is likely to hinder the propagation kinetics by reducing the rate of monomer diffusion toward the radical [10-13]. It is apparent how the reaction parameters governing the impact of the electrostatic interactions in polyelectrolyte systems, e.g. ionic strength and $\mathrm{pH}$, can be in principle used to tune the fundamental polymer and polymerization properties through their effect on the reaction kinetics.

In this paper we focus on the Am/AA aqueous copolymerization with the aim of investigating the kinetics of the system as a function of the electrostatic interactions as well as of the relevant parameters affecting their behavior. The kinetics of Am/AA radical copolymerization has been thoroughly investigated in the past decades with specific focus on the analysis of the composition behavior of the system. In this regard, studies on the estimation of the reactivity 
ratios for Am/AA have been carried out by several authors, and a large variety of data has been produced [14-18], whereas the main results have been summarized in a recent publication by Preusser et al. (cf. Fig. 1 in such paper) [19]. In particular, a comprehensive study on the effect of reaction parameters such as $\mathrm{pH}$ and ionic strength on the composition behavior of Am/AA copolymer system has been carried out by Rintoul et al., defining general trends for the estimated reactivity ratios as a function of the explored reaction conditions [17].

Generally, an increase of $r_{A m}=k_{p, A m / A m} / k_{p, A m / A A}$ and decrease of $r_{A A}=$ $k_{p, A A / A A} / k_{p, A A / A m}$ (with $k_{p, i / j}$ indicating the rate coefficient of the propagation between a radical chain with terminal unit of monomer $i$ with a monomer $j$ ) upon increasing $\mathrm{pH}$ or decreasing monomer concentration has been observed by the previous authors, pointing out the existence of a crossover $\mathrm{pH}$ for the reactivity ratios [17]. The possibility to control the copolymer composition of Am/AA by manipulating $\mathrm{pH}$ and ionic strength has been investigated by Paril et al., who identified specific reaction conditions to avoid composition drift [20]. Recent works by Riahinezhad et al. have been focused on the effect of reaction parameters governing the electrostatic interactions on the reactivity ratio estimations as well as on the copolymer microstructure (i.e., triad fractions) for Am/AA copolymerization [21]: particular attention has been dedicated to ionized AA and to the role played by the ionic strength [10].

In the last years, well-detailed characterizations of the composition behavior of radical copolymer systems have become possible thanks to the development and application of the socalled in-situ NMR technique, which has been employed to investigate in a comprehensive way the composition behavior of relevant polymer systems, including Am/AA [13,19,22-24]. Specifically, the collection of accurate composition data in all range of conversions has allowed to picture a broad overview of the behavior of Am/AA system at various degrees of ionization of AA and initial concentration / composition of the monomer mixture [19,22]. In the latest 
work on the topic, empirical equations for the terminal model reactivity ratios as a function of two reaction parameters, namely the degree of ionization of AA and the initial monomer concentration, have been proposed for the system [19].

In a previous work on the copolymerization of Am with the cationic monomer 2(acryloyloxyethyl)-trimethylammonium chloride (DMAEA-Q), a novel kinetic model to simulate the composition behavior of copolymer systems with ionic monomers was introduced [13]. The model is based on the concept of electrostatic screening of the repulsion forces coming from the charges on the reacting species during propagation due to the effect of an increase in the electrolyte concentration, induced either by working at higher concentration of the charged monomer or by adding salt to the polymerization system. Terminal model reactivity ratios function of the initial electrolyte concentration were evaluated based on a specifically-developed rate law for the propagation of the ionized monomer with an ionized terminal radical:

$$
k_{p}=\left(\frac{1}{k_{p}^{0}}+\frac{1}{k_{D} C_{E}^{\beta}}\right)^{-1}
$$

where $k_{p}^{0}$ is the propagation rate coefficient referred to the intrinsic kinetics of the reaction and $k_{D} C_{E}{ }^{\beta}$ the diffusion term accounting for the electrostatic repulsion between the charged species. Accordingly, the diffusion limitation to the overall propagation rate coefficient becomes weaker as the concentration of electrolyte increases (i.e., stronger electrostatic screening). As a result, the terminal model reactivity ratio corresponding to the ionized monomer $\left(r_{D M A E A-Q}\right)$ was defined as a function of the electrolyte concentration: this model feature was sufficient to reproduce the composition behavior of the system in a wide range of values of initial monomer concentration and ionic strength [13].

The primary target of the present work is to extend the previously-developed model to the Am/AA system: here, the situation is complicated by the presence of an ionizable monomer 
(AA) and thus by the need to consider its degree of dissociation as an additional model parameter. Although this feature was already included in the empirical model and reactivity ratio expressions proposed by Preusser et al. [19], the present work aims at developing model equations from the fundamental laws describing the reaction kinetics and the electrostatic effects. Moreover, the developed model is aimed at reproducing better the effect of the initial concentration of electrolyte on composition by decoupling the influence of monomer concentration from that of the addition of salt; to this purpose, the planned experimental analysis is focused on the copolymerization of Am with fully ionized AA and to the study of $\mathrm{NaCl}$ addition to polymerization experiments run at different initial monomer concentration. The model development and parameter estimation are carried out based on the experimental information already available about the system as well as on the results of targeted experiments by in-situ NMR aimed at elucidating the ionic strength effect on composition.

\section{Materials and Methods}

In-situ ${ }^{1} \mathrm{H}$ NMR experiments of copolymerization of fully ionized AA with Am are carried out to elucidate the composition effect of adding salt to the reaction in order to adjust the electrolyte concentration to higher values with respect to the ionic strength defined only by the presence of ionized monomer. Previous experiments by Preusser et al. revealed that it is possible to partly mimic the effect of concentration of the ionized monomer by salt addition [19]. The aims of the presented experimental campaign are to better understand this feature and to distinguish between the impact of electrostatic and non-electrostatic effects on the kinetics related to the concentration of the ionized monomer. Copolymerization reactions are carried out in deuterium oxide ( $\mathrm{D}_{2} \mathrm{O}$, Cambridge Isotope Laboratories, 99\% purity) and using NMR tubes (5 mm NMR tube, type 5UP, $178 \mathrm{~mm}$, ARMAR Chemicals) as reactors, which are inserted in an operating NMR spectrometer (Bruker UltraShield 500 MHz/54 mm magnet 
system). For each experiment, a solution of the two monomers Am (Sigma Aldrich, >99\% purity) and AA (anhydrous, Aldrich, 99\% purity, stabilized with 200 ppm of MEHQ inhibitor) and $\mathrm{NaOH}$ in $\mathrm{D}_{2} \mathrm{O}$ is prepared beforehand: the required amount of $\mathrm{NaCl}$ and of the radical initiator 2,2'-azobis(2-methylpropionamidine)-dihydrochloride (V-50, Acros Organics, 98\% purity) are added. The reaction mixture is degassed with $\mathrm{N}_{2}$ at $0^{\circ} \mathrm{C}$ for $5 \mathrm{~min}$ and about $0.6 \mathrm{~mL}$ of the reacting mixture are injected into the NMR tube, which is also stored at $0^{\circ} \mathrm{C}$. The total monomer concentration in the initial solution ( $w_{M, 0}$, weight fraction) ranges from $1 \%$ to $40 \%$, with initial composition $\left(f_{A, 0}\right.$, mole fraction of Am) equal to 0.5 , while the initial concentration of V-50 ( $w_{I, 0}$, weight fraction) is varied between $0.007 \%$ and $0.22 \%$ to counterbalance changes in the reaction rate. $\mathrm{NaCl}$ up to $15.9 \%$ ( $w_{S, 0}$, weight fraction) is added to the copolymerization reactions to increase the ionic strength of the solution. The reaction temperature is set to $50^{\circ} \mathrm{C}$ for all the experiments. The in-situ NMR procedure adopted for reaction monitoring, data acquisition, and determination of residual monomer composition as a function of conversion is identical to the one already discussed in a previous paper [13]: details of the monomer and polymer peak areas of each ${ }^{1} \mathrm{H}$ NMR spectrum used for these calculations are provided in Fig. S1 in the Supporting Information.

\section{Experimental results}

A first set of experiments has been carried out varying the initial monomer concentration, as detailed by runs 1-6 in Table 1. The results reported in Fig. 1 show that increasing the monomer concentration, and thus the amount of ionized monomer in the system, facilitates the incorporation of charged AA in the chains through the entire reaction. Consequently, the reaction mixture becomes richer in Am at each given monomer conversion, as already observed by Preusser et al. for Am/AA and similarly to the results obtained for the Am/DMAEA-Q system in our previous work $[13,19]$. Increasing the amount of ionized monomer enhances the 
electrostatic screening of the repulsion forces between the charged moieties of ionized AA units and explains the enhanced incorporation of such monomer in the chains.

Table 1. Experimental recipes for the copolymerization reactions of Am and AA at 50/50 initial molar ratio and $50^{\circ} \mathrm{C}$. The initial weight fraction of total monomer, added $\mathrm{NaCl}$, and $\mathrm{V}-50$ initiator (wt\%) as well as the initial concentration of electrolyte (mol kg-1) are indicated for each run.

\begin{tabular}{|c|c|c|c|c|}
\hline Run & $w_{M, 0}$ & $w_{S, 0}$ & $w_{I, 0}$ & $C_{E}$ \\
\hline 1 & $1 \%$ & - & $0.22 \%$ & 0.14 \\
\hline 2 & $5 \%$ & - & $0.22 \%$ & 0.70 \\
\hline 3 & $10 \%$ & - & $0.22 \%$ & 1.4 \\
\hline 4 & $20 \%$ & - & $0.022 \%$ & 2.8 \\
\hline 5 & $30 \%$ & - & $0.022 \%$ & 4.2 \\
\hline 6 & $40 \%$ & - & $0.0074 \%$ & 5.6 \\
\hline 7 & $1 \%$ & $15.9 \%$ & $0.022 \%$ & 5.6 \\
\hline 8 & $5 \%$ & $14.3 \%$ & $0.22 \%$ & 5.6 \\
\hline 9 & $10 \%$ & $12.2 \%$ & $0.022 \%$ & 5.6 \\
\hline 10 & $20 \%$ & $8.2 \%$ & $0.022 \%$ & 5.6 \\
\hline 11 & $30 \%$ & $4.1 \%$ & $0.0074 \%$ & 5.6 \\
\hline 12 & $1 \%$ & $1.6 \%$ & $0.22 \%$ & 0.70 \\
\hline 13 & $1 \%$ & $3.7 \%$ & $0.022 \%$ & 1.4 \\
\hline 14 & $1 \%$ & $7.8 \%$ & $0.022 \%$ & 2.8 \\
\hline 15 & $1 \%$ & $11.8 \%$ & $0.022 \%$ & 4.2 \\
\hline
\end{tabular}




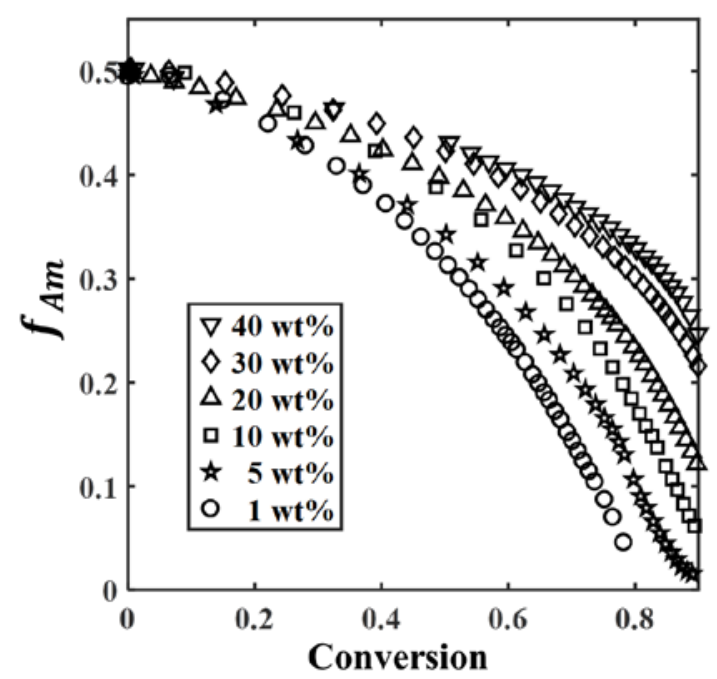

Fig. 1. Experimental results of residual monomer mixture composition as a function of conversion for the copolymerization of Am with fully ionized $\mathrm{AA}$ at $50^{\circ} \mathrm{C}$ corresponding to runs 1-6 in Table 1. The initial weight fraction of total monomer is detailed in the legend.

A second set of experiments has been carried out adjusting the initial ionic strength of experiments at different $w_{M, 0}$ by adding $\mathrm{NaCl}$, as detailed by runs 6-11 in Table 1 . Specifically, the same electrolyte concentration of the experiment at $w_{M, 0}$ equal to $40 \mathrm{wt} \%$ without added salt (run 6) has been set for all the other cases. The results are reported in Fig. 2. The comparison between Fig.s 1 and 2 shows a shift upward of the curves at low monomer concentration upon addition of $\mathrm{NaCl}$, consistently with the increased electrostatic screening that is achieved by rising the concentration of the ionized monomer. However, this effect can be only partly mimicked by salt addition, as revealed by the discrepancy between the curves in Fig. 2 at high conversion, and in agreement with the observations by Preusser et al. on the effect of $\mathrm{NaCl}$ addition [19]. This gap indicates that non-electrostatic effects also influence the composition behavior, most probably associated with the change in concentration of the water soluble monomers. 


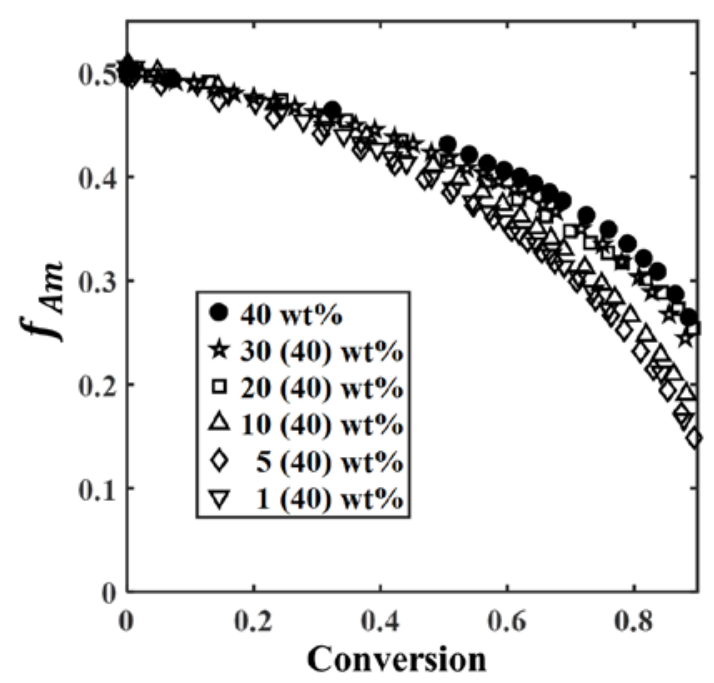

Fig. 2. Experimental results of residual monomer mixture composition as a function of conversion for the copolymerization of Am with fully ionized AA at $50^{\circ} \mathrm{C}$ corresponding to runs $6-11$ in Table 1 . The initial weight fraction of total monomer is detailed in the legend. In the experiments denoted by empty symbols, $\mathrm{NaCl}$ is added to increase the initial concentration of electrolyte to $5.6 \mathrm{~mol} \mathrm{~kg}{ }^{-1}$, corresponding to the case at $w_{M, 0}=40 \mathrm{wt} \%$ without added salt (the equivalent weight fraction of total monomer obtained after salt addition is reported within brackets in the legend).

The third set of experiments was aimed at decoupling the purely electrostatic effect of monomer concentration on the reaction kinetics from other monomer effects. Namely, experiments at the same initial monomer concentration (1 wt\%) but at increasing initial ionic strength by $\mathrm{NaCl}$ addition have been carried out, with reference to runs 1, 7, and 12-15 in Table 1. The gap between the curves in Fig. 3 reveals the impact of electrostatic effects on composition in a situation of low initial monomer concentration: such effect appears to be more relevant than the non-electrostatic one depicted by Fig. 2, especially at high conversion. 


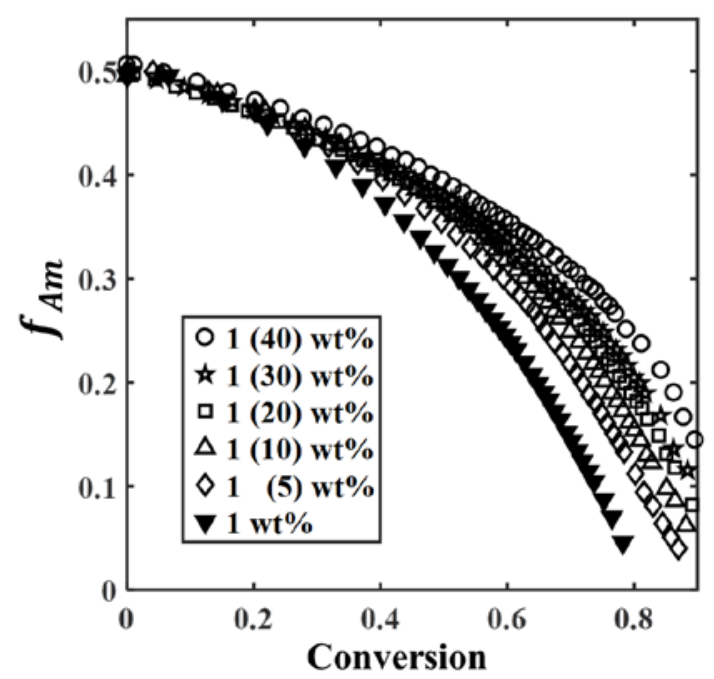

Fig. 3. Experimental results of residual monomer mixture composition as a function of conversion for the copolymerization of Am with fully ionized $\mathrm{AA}$ at $50^{\circ} \mathrm{C}$ corresponding to runs 1, 7, and 12-15 in Table 1. The initial weight fraction of total monomer is detailed in the legend. In the experiments denoted by empty symbols, $\mathrm{NaCl}$ is added to increase the initial concentration of electrolyte to higher values, corresponding to experiments at higher $w_{M, 0}$ without added salt (the equivalent weight fraction of total monomer obtained after salt addition is reported within brackets in the legend).

To better understand the effect of the two contributions laying behind an increase in monomer concentration (i.e., non-electrostatic and electrostatic), the previously discussed results of runs 1-2, 5-6, and 11-12 have been reported again in Fig. 4. Runs 1-2 and 12 show that the effect of increasing monomer concentration on composition at low $w_{M, 0}$ values (i.e., from $1 \mathrm{wt} \%$ to $5 \mathrm{wt} \%$ ) is relevant and can be mimicked almost entirely by adding $\mathrm{NaCl}$ to rise the ionic strength: in this condition, the electrostatic features are mostly responsible for the observed kinetic effects coming from an increase in monomer concentration. On the other hand, runs 5-6 and 11 reveals that at high $w_{M, 0}$ values (i.e., $30 \mathrm{wt} \%$ to $40 \mathrm{wt} \%$ ) the change in 
composition behavior can be attributed solely to non-electrostatic effects of changing monomer concentration, since increasing the electrolyte concentration does not modify the composition curve. As already mentioned before, the impact of these non-electrostatic effects on the composition behavior is generally smaller than that of the electrostatic effects; nevertheless, it becomes relevant - and even dominant - at higher monomer concentration.

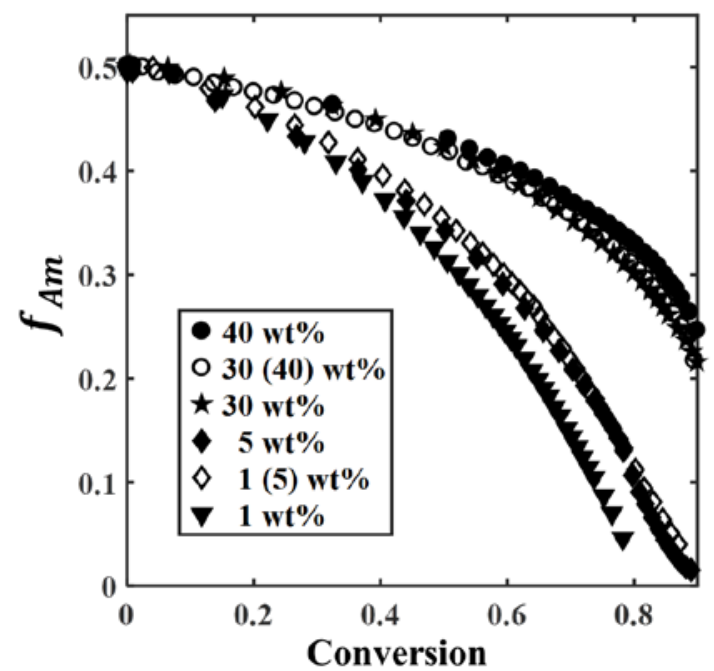

Fig. 4. Experimental results of residual monomer mixture composition as a function of conversion for the copolymerization of Am with fully ionized AA at $50^{\circ} \mathrm{C}$ corresponding to the runs 1,2,12 and 5,6,11 in Table 1 . The initial weight fraction of total monomer is detailed in the legend. In the experiments denoted by empty symbols, $\mathrm{NaCl}$ is added to increase the initial concentration of electrolyte to higher values, corresponding to experiments at higher $w_{M, 0}$ without added salt (the equivalent weight fraction of total monomer obtained after salt addition is reported within brackets in the legend).

The reported experimental results show quite clearly the limitations involved in the reproduction of the monomer concentration effect on the composition behavior by salt addition, in particular at different initial monomer contents. Moreover, their consistency with the results 
obtained by Preusser et al. is demonstrated by Fig. S2 in the Supporting Information, where experimental data collected by the two groups at the same monomer and electrolyte concentration are compared [19].

\section{Model Development}

A copolymerization model suitable to predict the composition behavior of Am/AA is proposed, with focus on the development of relationships providing the reactivity ratio values as a function of monomer and electrolyte concentration. Such functionality is introduced accounting for the effect of electrostatic interactions on the propagation kinetics into the conventional copolymerization equations. In order to differentiate the reactivity of ionized and non-ionized forms of acrylic acid, a ternary polymer system is considered, with Am, ionized AA, and non-ionized AA (the last two indicated as $\mathrm{A}^{-}$and HA, respectively) as monomers.

As an improvement to our previous model for Am/DMAEA-Q copolymerization [13], a penultimate unit copolymerization model is considered to describe the effect of the electrostatic interactions on the propagation kinetics. This choice is supported by the following consideration about the propagation reaction in the polymerization of ionized AA. When the approaching monomer is sufficiently close to the terminal radical unit to generate a relevant electrostatic repulsion, the penultimate unit in the radical chain would also be sufficiently close to the approaching monomer to expect also electrostatic repulsion between the charges on monomer and penultimate unit in the radical chain. In comparison with the previous work about Am/DMAEA-Q [13], where the penultimate unit effect on the electrostatic interactions could be neglected, the repeating AA units in this copolymer exhibit a charge-bearing side chain, which is much shorter than DMAEA-Q. As a consequence, the minimum distance between the charges on monomer and penultimate radical unit in the transition state of AA propagation is much shorter compared to the distance between the charges on monomer and terminal radical 
unit in DMAEA-Q, as detailed in Fig. S3 for AA and DMAEA-Q oligomers obtained by quantum chemistry calculations [25-28].

The kinetic scheme defining the propagation reactions of the ternary system assuming a penultimate unit model involves 27 reactions, which can be written as follows along with the corresponding reaction rates $\left(R_{P}\right)$ :

$$
\begin{aligned}
& R_{i j}+M_{k} \stackrel{k_{p, i j k}}{\longrightarrow} R_{j k} \\
& R_{P, i j k}=k_{p, i j k} R_{i j} M_{k}
\end{aligned}
$$

where the subscripts $i, j$, and $k$ denote $\mathrm{Am}, \mathrm{A}^{-}$, and HA units, $R_{i j}$ is a radical chain with terminal unit of $j$ and penultimate unit of $i$, and $M_{k}$ is a monomer unit of $k$. It should be noted that only chain-end propagation is considered in this study: although secondary reactions are known to occur for both Am and AA polymerization [29,30], the effect of mid-chain radical propagation on copolymer composition is negligible [13].

Pseudo-binary system. The correlation between the concentrations of ionized and nonionized forms of AA can be expressed by the degree of dissociation of the monomer $\left(\alpha_{M}\right)$, which is defined as:

$$
\alpha_{M}=\frac{\left[A^{-}\right]}{[H A]+\left[A^{-}\right]}
$$

Since AA is a weak acid, its degree of dissociation in aqueous solution can be adjusted by adding a strong base, e.g. $\mathrm{NaOH}$ : in this case, the initial monomer dissociation can be quickly estimated as the ratio between the concentrations of added base, $[B]_{0}$, and of initial monomer, $[A A]_{0}$. As the reaction starts and some polymer is formed, another degree of dissociation must be considered because AA monomer and AA polymer units exhibit different values of equilibrium constant of dissociation, $K_{a}\left(p K_{a, M}\right.$ of 4.2 for the free monomer and $p K_{a, P}$ of 4.75 for the polymer units) [10]. Accordingly, a polymer dissociation constant is defined: 


$$
\alpha_{P}=\frac{\left[A^{-}\right]_{P}}{[H A]_{P}+\left[A^{-}\right]_{P}}
$$

where $\left[A^{-}\right]_{P}$ and $[H A]_{P}$ are the concentrations of total monomer units of ionized and nonionized AA in the polymer chains, respectively. It should be noted that a constant $p K_{a, P}$ value has been adopted for all polymer units, which implies some important assumptions about the polymer dissociation constant aimed at simplifying the model. Namely, the resulting value of $\alpha_{P}$ is independent of neither the polymer chain length nor the ionization state of the adjacent units. Moreover, it is applied to both radical and non-radical units (specifically, the $p K_{a, P}$ value adopted is referred to non-reactive systems). A more accurate characterization of the ionization state of the polymer units in the chains should also take into account the dependence of $p K_{a, P}$ on the degree of ionization of the polymer [31]. The parameters $\alpha_{M}$ and $\alpha_{P}$ can be expressed more conveniently as a function of the corresponding $K_{a}$ and $\mathrm{pH}$ values: the latter can be in turn evaluated from the monomer conversion and the initial degree of dissociation of AA, as detailed in the Supporting Information.

Equations 4 and 5 correlate the concentrations of ionized and non-ionized AA, respectively, for free monomer and polymer units: they can be used to express the radical and monomer concentrations corresponding to the ternary system $\left(R_{i j}\right.$ and $M_{k}$ with $\left.i, j, k=\mathrm{Am}, \mathrm{A}^{-}, \mathrm{HA}\right)$ as a function of the radical and monomer concentrations corresponding to the binary system $\left(R_{i j}\right.$ and $M_{k}$ with $\left.i, j, k=\mathrm{Am}, \mathrm{AA}\right)$ and of the degrees of dissociation, as detailed in the Supporting Information. The combination of these expressions with the reaction rates defined by Equation 3 leads to the definition of the kinetic scheme of a pseudo-binary system comprehensive of 8 reactions, which are reported in Table 2 along with the corresponding pseudo-binary propagation rate coefficients. The derivation of the pseudo-binary propagation rates and rate coefficients is detailed again in the Supporting Information. 
Table 2. Reaction scheme and rate coefficients of the propagation reactions in Am/AA copolymerization considering a pseudo-binary system.

\begin{tabular}{|c|c|}
\hline Reaction & Pseudo-binary propagation rate coefficient \\
\hline $\begin{array}{c}R_{A m / A m}+M_{A m} \rightarrow \\
R_{A m / A m}\end{array}$ & $k_{p, A m / A m / A m}$ \\
\hline$R_{A m / A m}+M_{A A} \rightarrow R_{A m / A A}$ & $k_{p, A m / A m / A A}=k_{p, A m / A m / A-} \alpha_{M}+k_{p, A m / A m / H A}\left(1-\alpha_{M}\right)$ \\
\hline$R_{A m / A A}+M_{A m} \rightarrow R_{A A / A m}$ & $k_{p, A m / A A / A m}=k_{p, A m / A-/ A m} \alpha_{P}+k_{p, A m / H A / A m}\left(1-\alpha_{P}\right)$ \\
\hline$R_{A m / A A}+M_{A A} \rightarrow R_{A A / A A}$ & $\begin{array}{c}k_{p, A m / A A / A A}=k_{p, A m / A-/ A-} \alpha_{P} \alpha_{M}+k_{p, A m / A-/ H A} \alpha_{P}(1- \\
\left.\alpha_{M}\right)+k_{p, A m / H A / A-}\left(1-\alpha_{P}\right) \alpha_{M}+k_{p, A m / H A / H A}(1- \\
\left.\alpha_{P}\right)\left(1-\alpha_{M}\right)\end{array}$ \\
\hline $\begin{array}{c}R_{A A / A m}+M_{A m} \rightarrow \\
R_{A m / A m}\end{array}$ & $k_{p, A A / A m / A m}=k_{p, A-/ A m / A m} \alpha_{P}+k_{p, H A / A m / A m}\left(1-\alpha_{P}\right)$ \\
\hline$R_{A A / A m}+M_{A A} \rightarrow R_{A m / A m}$ & $\begin{array}{c}k_{p, A A / A m / A A}=k_{p, A-/ A m / A-} \alpha_{P} \alpha_{M}+k_{p, A-/ A m / H A} \alpha_{P}(1- \\
\left.\alpha_{M}\right)+k_{p, H A / A m / A-}\left(1-\alpha_{P}\right) \alpha_{M}+k_{p, H A / A m / H A}(1- \\
\left.\alpha_{P}\right)\left(1-\alpha_{M}\right)\end{array}$ \\
\hline$R_{A A / A A}+M_{A m} \rightarrow R_{A A / A m}$ & $\begin{array}{l}k_{p, A A / A A / A m}=k_{p, A-/ A-/ A m}\left(\alpha_{P}\right)^{2}+k_{p, A-/ H A / A m} \alpha_{P}(1- \\
\left.\alpha_{P}\right)+k_{p, H A / A-/ A m}\left(1-\alpha_{P}\right) \alpha_{P}+k_{p, H A / H A / A m}\left(1-\alpha_{P}\right)^{2}\end{array}$ \\
\hline$R_{A A / A A}+M_{A A} \rightarrow R_{A A / A A}$ & $\begin{array}{c}k_{p, A A / A A / A A}=k_{p, A-/ A-/ A-}\left(\alpha_{P}\right)^{2} \alpha_{M}+ \\
k_{p, A-/ A-/ H A}\left(\alpha_{P}\right)^{2}\left(1-\alpha_{M}\right)+k_{p, A-/ H A / A-} \alpha_{P}(1- \\
\left.\alpha_{P}\right) \alpha_{M}+k_{p, A-/ H A / H A} \alpha_{P}\left(1-\alpha_{P}\right)\left(1-\alpha_{M}\right)+ \\
k_{p, H A / A-/ A-}\left(1-\alpha_{P}\right) \alpha_{P} \alpha_{M}+k_{p, H A / A-/ H A}\left(1-\alpha_{P}\right) \alpha_{P}(1- \\
\alpha_{M}+k_{p, H A / H A / A-}\left(1-\alpha_{P}\right)^{2} \alpha_{M}+k_{p, H A / H A / H A}(1- \\
\left.\alpha_{P}\right)^{2}\left(1-\alpha_{M}\right)\end{array}$ \\
\hline
\end{tabular}

Electrostatic interactions. The pseudo-binary system described by the equations in Table 2 includes 8 pseudo-binary propagation rate coefficients which are function of the 27 propagation rate coefficients of the original ternary system. Among those parameters, those referring to the reactions involving a charged monomer $\left(\mathrm{A}^{-}\right)$and at least one charged polymer unit among terminal and penultimate ones are likely to exhibit the effect of the electrostatic interactions on the propagation kinetics. Such effect is accounted for through the previously developed rate 
law in Equation 1, which expresses the propagation rate coefficient as a function of the initial electrolyte concentration $\left(C_{E}\right)$. The latter quantity can be estimated as:

$$
C_{E}=2\left(\frac{x_{A A, 0} w_{M, 0} \alpha}{M W_{A A}}+\frac{w_{S, 0}}{M W_{S}}\right)
$$

where $x_{A A, 0}$ is the initial weight fraction of AA with respect to the monomer mixture, $w_{M, 0}$ and $w_{S, 0}$ the initial weight fractions of total monomer and added salt (considering a 1:1 electrolyte, e.g. $\mathrm{NaCl}$ ), respectively, and $M W_{A A}$ and $M W_{S}$ the molecular weights of $\mathrm{AA}$ and salt, respectively. The electrolyte concentration is provided by Equation 6 as moles of total electrolyte per kg of solution.

In comparison with the previously investigated Am/DMAEA-Q system, the assumption of a penultimate unit effect on the electrostatic interactions increases to five the number of propagation rate coefficients which are function of the electrolyte concentration:

$$
\begin{aligned}
k_{p, A-/ A-/ A-} & =\left(\frac{1}{k_{p, A-/ A-/ A-}^{0}}+\frac{1}{k_{D, C C C} C_{E}^{\beta_{C C C}}}\right)^{-1} \\
k_{p, A-/ H A / A-} & =\left(\frac{1}{k_{p, A-/ H A / A-}^{0}}+\frac{1}{k_{D, C X C} C_{E}^{\beta_{C X C}}}\right)^{-1} \\
k_{p, A-/ A m / A-} & =\left(\frac{1}{k_{p, A-/ A m / A-}^{0}}+\frac{1}{k_{D, C X C} C_{E}^{\beta_{C X C}}}\right)^{-1} \\
k_{p, H A / A-/ A-} & \left(\frac{1}{k_{p, H A / A-/ A-}^{0}}+\frac{1}{k_{D, X C C} C_{E}^{\beta_{X C C}}}\right)^{-1} \\
k_{p, A m / A-/ A-} & =\left(\frac{1}{k_{p, A m / A-/ A-}^{0}}+\frac{1}{k_{D, X C C} C_{E}^{\beta_{X C C}}}\right)^{-1} \\
&
\end{aligned}
$$


In Equations 7-11, five different intrinsic kinetics terms $\left(k_{p}^{0}\right)$ are defined: the superscript “0” indicates that the parameter is not function of the electrolyte concentration. The remaining 22 rate coefficients of the penultimate unit model coincide with the corresponding intrinsic kinetics terms, thus they also do not exhibit any dependence on $C_{E}$.

Under the assumption of electrostatic interaction effects not influenced by the nature of the non-ionized monomer but only by the number and position of the charged units involved in the propagation reaction, only three different cases have to be considered in the equations above regarding the diffusion terms: (i) monomer and terminal radical units are charged (parameters $k_{D, X C C}$ and $\beta_{X C C}$ ); (ii) monomer and penultimate radical units are charged (parameters $k_{D, C X C}$ and $\beta_{C X C}$ ); (iii) monomer and both units in the radical chain are charged (parameters $k_{D, C C C}$ and $\left.\beta_{C C C}\right)$. In the subscripts, "C" stands for a charged monomer or polymer unit $\left(\mathrm{A}^{-}\right)$and " $\mathrm{X}$ " for a non-charged unit (Am or HA). Overall, the model involves 27 parameters of the intrinsic kinetics and 6 parameters of the electrostatic interactions.

Implicit penultimate unit model. As anticipated, this specific propagation model has been considered to improve the description of the effect of the electrostatic interactions. Therefore, it is applied only in the case of electrostatically interacting species (charged monomer and monomer repeating units), while a simpler terminal model has been applied to describe the intrinsic kinetics terms of the propagation rate coefficient. This way, a significant reduction of the number of model parameters is achieved, as detailed in Table 3. By combining the pseudobinary propagation rate coefficients in Table 2 with the expressions defined in Table 3 as well as by Equations 7-11, an implicit penultimate model of copolymerization is finally obtained, where the penultimate unit effect is considered only for the electrostatic interactions but not for the intrinsic kinetics of the system:

$$
k_{p, A m / A m / A m}=k_{p, A m / A m}^{0}
$$




$$
\begin{aligned}
& k_{p, A m / A m / A A}=k_{p, A m / A-}^{0} \alpha_{M}+k_{p, A m / H A}^{0}\left(1-\alpha_{M}\right) \\
& k_{p, A m / A A / A m}=k_{p, A-/ A m}^{0} \alpha_{P}+k_{p, H A / A m}^{0}\left(1-\alpha_{P}\right)
\end{aligned}
$$

$$
\begin{aligned}
k_{p, A m / A A / A A}= & \left(\frac{1}{k_{p, A-/ A-}^{0}}+\frac{1}{k_{D, X C C} C_{E}^{\beta_{X C C}}}\right)^{-1} \alpha_{P} \alpha_{M}+k_{p, A-/ H A}^{0} \alpha_{P}\left(1-\alpha_{M}\right) \\
& +k_{p, H A / A-}^{0}\left(1-\alpha_{P}\right) \alpha_{M}+k_{p, H A / H A}^{0}\left(1-\alpha_{P}\right)\left(1-\alpha_{M}\right) \\
k_{p, A A / A m / A m}= & k_{p, A m / A m}^{0} \alpha_{P}+k_{p, A m / A m}^{0}\left(1-\alpha_{P}\right)=k_{p, A m / A m}^{0}
\end{aligned}
$$

$$
\begin{aligned}
k_{p, A A / A m / A A}= & \left(\frac{1}{k_{p, A m / A-}^{0}}+\frac{1}{k_{D, C X C} C_{E}^{\beta_{C X C}}}\right)^{-1} \alpha_{P} \alpha_{M}+k_{p, A m / H A}^{0} \alpha_{P}\left(1-\alpha_{M}\right) \\
& +k_{p, A m / A-}^{0}\left(1-\alpha_{P}\right) \alpha_{M}+k_{p, A m / H A}^{0}\left(1-\alpha_{P}\right)\left(1-\alpha_{M}\right) \\
& =\left(\frac{1}{k_{p, A m / A-}^{0}}+\frac{1}{k_{D, C X C} C_{E}^{\beta_{C X C}}}\right)^{-1} \alpha_{P} \alpha_{M}+k_{p, A m / H A}^{0}\left(1-\alpha_{M}\right) \\
& +k_{p, A m / A-}^{0}\left(1-\alpha_{P}\right) \alpha_{M}
\end{aligned}
$$

$$
k_{p, A A / A A / A m}=k_{p, A-/ A m}^{0} \alpha_{P}+k_{p, H A / A m}^{0}\left(1-\alpha_{P}\right)
$$

$$
\begin{aligned}
k_{p, A A / A A / A A}= & \left.\frac{1}{k_{p, A-/ A-}^{0}}+\frac{1}{k_{D, C C C} C_{E}^{\beta C C C}}\right)^{-1}\left(\alpha_{P}\right)^{2} \alpha_{M}+k_{p, A-/ H A}^{0} \alpha_{P}\left(1-\alpha_{M}\right) \\
& +\left(\frac{1}{k_{p, H A / A-}^{0}}+\frac{1}{k_{D, C X C} C_{E}^{\beta_{C X C}}}\right)^{-1} \alpha_{P}\left(1-\alpha_{P}\right) \alpha_{M} \\
& +k_{p, H A / H A}^{0}\left(1-\alpha_{P}\right)\left(1-\alpha_{M}\right) \\
& +\left(\frac{1}{k_{p, A-/ A-}^{0}}+\frac{1}{k_{D, X C C} C_{E}^{\beta_{X C C}}}\right)^{-1}\left(1-\alpha_{P}\right) \alpha_{P} \alpha_{M} \\
& +k_{p, H A / A-}^{0}\left(1-\alpha_{P}\right)^{2} \alpha_{M}
\end{aligned}
$$


Table 3. Simplification of the penultimate unit model parameters (left column) after neglecting the penultimate unit effect on the intrinsic kinetics of propagation reactions (center column) and after the definition of the binary system reactivity ratios (right column).

\begin{tabular}{|c|c|c|}
\hline Penultimate unit model & Terminal model & Reactivity ratios \\
\hline$k_{p, A m / A m / A m}=k_{p, A-/ A m / A m}=k_{p, H A / A m / A m}$ & $=k_{p, A m / A m}^{0}$ & \\
\hline$k_{p, A m / H A / H A}=k_{p, A-/ H A / H A}=k_{p, H A / H A / H A}$ & $=k_{p, H A / H A}^{0}$ & \\
\hline$k_{p, A-/ A-/ A-}^{0}=k_{p, H A / A-/ A-}^{0}=k_{p, A m / A-/ A-}^{0}$ & $=k_{p, A-/ A-}^{0}$ & $S_{A-/ H A}=\frac{k_{p, A-/ A-}^{0}}{k_{p, H A / H A}^{0}}$ \\
\hline$k_{p, A-/ A m / H A}=k_{p, H A / A m / H A}=k_{p, A m / A m / H A}$ & $=k_{p, A m / H A}^{0}$ & $r_{A m / H A}=\frac{k_{p, A m / A m}^{0}}{k_{p, A m / H A}^{0}}$ \\
\hline$k_{p, A-/ H A / A m}=k_{p, H A / H A / A m}=k_{p, A m / H A / A m}$ & $=k_{p, H A / A m}^{0}$ & $r_{H A / A m}=\frac{k_{p, H A / H A}^{0}}{k_{p, H A / A m}^{0}}$ \\
\hline$k_{p, A-/ A m / A-}^{0}=k_{p, A m / A m / A-}=k_{p, H A / A m / A-}$ & $=k_{p, A m / A-}^{0}$ & $r_{A m / A-}=\frac{k_{p, A m / A m}^{0}}{k_{p, A m / A-}^{0}}$ \\
\hline$k_{p, A-/ A-/ A m}=k_{p, A m / A-/ A m}=k_{p, H A / A-/ A m}$ & $=k_{p, A-/ A m}^{0}$ & $r_{A-/ A m}=\frac{k_{p, A-/ A-}^{0}}{k_{p, A-/ A m}^{0}}$ \\
\hline$k_{p, A-/ H A / A-}^{0}=k_{p, H A / H A / A-}=k_{p, A m / H A / A-}$ & $=k_{p, H A / A-}^{0}$ & $r_{H A / A-}=\frac{k_{p, H A / H A}^{0}}{k_{p, H A / A-}^{0}}$ \\
\hline$k_{p, A-/ A-/ H A}=k_{p, H A / A-/ H A}=k_{p, A m / A-/ H A}$ & $=k_{p, A-/ H A}^{0}$ & $r_{A-/ H A}=\frac{k_{p, A-/ A-}^{0}}{k_{p, A-/ H A}^{0}}$ \\
\hline
\end{tabular}

The model involves now only 9 parameters of the intrinsic kinetics and 6 parameters of the electrostatic interactions. It is worth noting again that Equations 12-19 correspond to the set of propagation rate coefficients of a typical penultimate unit model, even though the simplification introduced in this paragraph has reduced the number of model parameters. 
Reactivity ratios. In the penultimate unit model of copolymerization, the conventional Mayo-Lewis equation of a terminal model can be applied to express the instantaneous copolymer composition, $F_{A m}$, as a function of the residual monomer mixture composition, $f_{A m}$, providing that pseudo-terminal-model reactivity ratios $\left(\overline{r_{A m}}\right.$ and $\left.\overline{r_{A A}}\right)$ are introduced:

$$
\begin{aligned}
& F_{A m}=\frac{\overline{r_{A m}} f_{A m}^{2}+f_{A m} f_{A A}}{\overline{r_{A m}} f_{A m}^{2}+2 f_{A m} f_{A A}+\overline{r_{A A}} f_{A A}^{2}} \\
& \overline{r_{A m}}=r_{A m}^{\prime}\left(\frac{f_{A m} r_{A m}+f_{A A}}{f_{A m} r_{A m}^{\prime}+f_{A A}}\right) \\
& \overline{r_{A A}}=r_{A A}^{\prime}\left(\frac{f_{A A} r_{A A}+f_{A m}}{f_{A A} r_{A A}^{\prime}+f_{A m}}\right)
\end{aligned}
$$

In Equations 21-22, the pseudo-terminal-model reactivity ratios are function of monomer composition as well as of the following 4 reactivity ratios of the penultimate unit model:

$$
\begin{gathered}
r_{A m}=\frac{k_{p, A m / A m / A m}}{k_{p, A m / A m / A A}} \\
r_{A A}=\frac{k_{p, A A / A A / A A}}{k_{p, A A / A A / A m}} \\
r_{A m}^{\prime}=\frac{k_{p, A A / A m / A m}}{k_{p, A A / A m / A A}} \\
r_{A A}^{\prime}=\frac{k_{p, A m / A A / A A}}{k_{p, A m / A A / A m}}
\end{gathered}
$$

Introducing the propagation rate coefficients from Equations 12-19 in the reactivity ratios defined by Equations 23-26, the copolymerization model is completely defined. By solving the Skeist formula [32], where the instantaneous copolymer composition is defined as in Equation 
20, the conversion-evolution of residual monomer composition can be evaluated as a function of $C_{E}$. More precisely, both pseudo-terminal model reactivity ratios defined by Equations 2122 are dependent on the initial monomer and electrolyte concentration thanks to the assumption of a penultimate unit effect on the electrostatic interactions. This model feature is in agreement with Preusser et al., who revealed the need of introducing two terminal model reactivity ratios function of the monomer concentration in order to fit the composition data of Am copolymerization with fully ionized AA (cf. Fig. 11 in the reference paper) [19].

In Equations 12-19, the 9 model parameters defining the intrinsic kinetics of propagation listed in Table 3 can be expressed more conveniently as a function of 7 reactivity ratios, which correspond to the 3 binary copolymer systems $\mathrm{Am} / \mathrm{HA}, \mathrm{Am} / \mathrm{A}^{-}$, and $\mathrm{A}^{-} / \mathrm{HA}$, as detailed again in Table 3. Specifically, the rate coefficients of cross-propagation reactions are referred to the corresponding homo-propagation rate coefficient involving the same radical; additionally, the ratio of homo-propagation rate coefficients of $\mathrm{A}^{-}$and $\mathrm{HA}, s_{A-/ H A}$, is considered. After introducing the newly-defined reactivity ratios in the model equations, the penultimate unit model reactivity ratios in Equations 23-26 assume their final form:

$$
r_{A m}=\frac{1}{\left(\frac{\alpha_{M}}{r_{A m / A-}}+\frac{1-\alpha_{M}}{r_{A m / H A}}\right)}
$$




$$
\begin{aligned}
& r_{A A} \\
& =\frac{\left(1+\frac{k_{p, H A / H A}^{0} s_{A-/ H A}}{k_{D, C C C} C_{E}^{\beta_{C C C}}}\right)^{-1}\left(\alpha_{P}\right)^{2} \alpha_{M}+\frac{\alpha_{P}\left(1-\alpha_{M}\right)}{r_{A-/ H A}}}{\frac{\alpha_{P}}{r_{A-/ A m}}+\frac{\left(1-\alpha_{P}\right)}{r_{H A / A m} S_{A-/ H A}}} \\
& +\frac{\left(r_{H A / A-} s_{A-/ H A}+\frac{k_{p, H A / H A}^{0} s_{A-/ H A}}{k_{D, C X C} C_{E}^{\beta_{C X C}}}\right)^{-1} \alpha_{P}\left(1-\alpha_{P}\right) \alpha_{M}+\frac{\left(1-\alpha_{P}\right)\left(1-\alpha_{M}\right)}{s_{A-/ H A}}}{\frac{\alpha_{P}}{r_{A-/ A m}}+\frac{\left(1-\alpha_{P}\right)}{r_{H A / A m} s_{A-/ H A}}} \\
& +\frac{\left(1+\frac{k_{p, H A / H A}^{0} s_{A-/ H A}}{k_{D, X C C} C_{E}^{\beta_{X C C}}}\right)^{-1}\left(1-\alpha_{P}\right) \alpha_{P} \alpha_{M}+\frac{\left(1-\alpha_{P}\right)^{2} \alpha_{M}}{r_{H A / A-} S_{A-/ H A}}}{\frac{\alpha_{P}}{r_{A-/ A m}}+\frac{\left(1-\alpha_{P}\right)}{r_{H A / A m} S_{A-/ H A}}} \\
& r_{A m}^{\prime}=\frac{1}{\left(r_{A m / A-}+\frac{k_{p, A m / A m}^{0}}{k_{D, C X C} C_{E}^{\beta_{C X C}}}\right)^{-1} \alpha_{P} \alpha_{M}+\frac{1-\alpha_{M}}{r_{A m / H A}}+\frac{\left(1-\alpha_{P}\right) \alpha_{M}}{r_{A m / A-}}} \\
& r_{A A}^{\prime}=\frac{\left(1+\frac{k_{p, H A / H A}^{0} s_{A-/ H A}}{k_{D, X C C} C_{E}^{\beta_{X C C}}}\right)^{-1} \alpha_{P} \alpha_{M}+\frac{\alpha_{P}\left(1-\alpha_{M}\right)}{r_{A-/ H A}}}{\frac{\alpha_{P}}{r_{A-/ A m}}+\frac{1-\alpha_{P}}{r_{H A / A m} s_{A-/ H A}}} \\
& +\frac{\frac{\left(1-\alpha_{P}\right) \alpha_{M}}{r_{H A / A-} s_{A-/ H A}}+\frac{\left(1-\alpha_{P}\right)\left(1-\alpha_{M}\right)}{s_{A-/ H A}}}{\frac{\alpha_{P}}{r_{A-/ A m}}+\frac{1-\alpha_{P}}{r_{H A / A m} s_{A-/ H A}}}
\end{aligned}
$$

Equations 27-30 involve two absolute rate coefficients of propagation, $k_{p, A m / A m}^{0}$ and $k_{p, H A / H A}^{0}$, which have been already determined by pulsed-laser polymerization (PLP) experiments [33,34]: the corresponding equations function of initial monomer concentration and conversion have been implemented in the model. It should be noted that these two parameters correspond to propagation steps which are not affected by electrostatic interactions. Moreover, Equations 27-30 are function of 7 binary-system reactivity ratios. Including also the 6 parameters of the electrostatic interactions introduced before, the final model defined by 
Equations 20-22 and 27-30 involves overall 13 unknown parameters, which have to be estimated from a large enough set of experimental data.

\section{Model Results and Discussion}

Given the large number of adjustable model parameters (13), the following approach has been applied for their estimation:

- the values of the two reactivity ratios corresponding to the binary system Am/HA have been taken from the study of the copolymerization of Am with non-ionized AA carried out by Preusser et al. [22]: $r_{A m / H A}=0.55$ and $r_{H A / A m}=1.24$ (referred to as $r_{A m}$ and $r_{A A}$ in the cited work, respectively);

- the remaining 11 parameters have been estimated by fitting the model predictions to relevant experimental information, which includes composition data obtained by in-situ NMR in this work and by Preusser et al. [19] as well as data of propagation kinetics estimated by PLP combined with size-exclusion chromatography (PLP-SEC) [35].

- finally, the reliability of the developed model as well as of the estimated model parameters has been tested by prediction of experimental data from additional experiments not used for the parameter evaluation.

Reduced propagation rate coefficient of fully ionized AA. Equation 19 can be used to evaluate the propagation rate coefficient of fully ionized AA, which is given by:

$$
k_{p, A A, \alpha=1}=k_{p, A-/ A-/ A-}=\left(\frac{1}{k_{p, A-/ A-}^{0}}+\frac{1}{k_{D, C C C} C_{E}^{\beta_{C C C}}}\right)^{-1}
$$

A reduced rate coefficient at $\alpha=1$ can be defined dividing Equation 31 by the propagation rate constant of non-ionized AA, according to the definition given by Lacik et al.[35]: 


$$
\frac{k_{p, A A, \alpha=1}}{k_{p, A A, \alpha=0}}=\frac{\left(\frac{1}{k_{p, A-/ A-}^{0}}+\frac{1}{k_{D, C C C} C_{E}^{\beta C C C}}\right)^{-1}}{k_{p, H A / H A}^{0}}=\left(\frac{1}{s_{A-/ H A}}+\frac{k_{p, H A / H A}^{0}}{k_{D, C C C} C_{E}^{\beta_{C C C}}}\right)^{-1}
$$

The unknown model parameters in the right-hand side of Equation $32\left(s_{A-/ H A}, k_{D, C C C}\right.$, and $\beta_{C C C}$ ) can be estimated by fitting the experimental values of the reduced propagation rate coefficient of fully ionized MAA as a function of monomer concentration reported by Lacik et al. [35] . Although these data have been evaluated for MAA, in the same work the authors demonstrated that the reduced propagation rate coefficient as a function of $\alpha$ is independent of the type of monomer (AA or MAA). The selected parameter estimation approach is detailed hereinafter:

1. The model parameter $\beta_{C C C}$ has been first estimated taking advantage of the following modified log-log version of Equation 32:

$$
\log \left(\frac{k_{p, A A, \alpha=1}}{k_{p, A A, \alpha=0}}\right)=-\log \left(\frac{1}{s_{A-/ H A}}+\frac{k_{p, H A / H A}^{0}}{k_{D, C C C} C_{E}^{\beta_{C C C}}}\right)
$$

Considering the limiting case of negligible electrolyte concentration (i.e., $C_{E} \rightarrow 0$, corresponding to very low monomer concentration in absence of added salt), the diffusion term is dominant over the intrinsic kinetics term, so that the latter can be neglected. Accordingly:

$$
\left.\log \left(\frac{k_{p, A A, \alpha=1}}{k_{p, A A . \alpha=0}}\right)\right|_{C_{E} \rightarrow 0}=-\log \left(k_{p, H A / H A}^{0}\right)+\log \left(k_{D, C C C}\right)+\beta_{C C C} \log \left(C_{E}\right)
$$

In the previous equation, the parameter $\beta_{C C C}$ is the slope of the logarithm of the reduced propagation rate coefficient as a function of the logarithm of the electrolyte or monomer concentration at very low values of $C_{E}$ (or $w_{M, 0}$ ). The corresponding experimental values are shown in the double-logarithmic plot of Fig. 5a: from the two values of the reduced propagation rate coefficient at the lowest monomer concentrations (i.e., $5 \%$ and $20 \%$ ), $\beta_{C C C}=1.26$ is evaluated. 
2. The remaining two parameters are then estimated by fitting Equation 32 to the same experimental data as shown in Fig. 5b. Final values of $k_{D, C C C}=1.02 \cdot 10^{4} \mathrm{~L} \mathrm{~mol}^{-1} \mathrm{~s}^{-1}\left(\mathrm{~kg} \mathrm{~mol}^{-}\right.$ $\left.{ }^{1}\right)^{\beta}$ ) and $s_{A-/ H A}=1.33$ have been obtained.
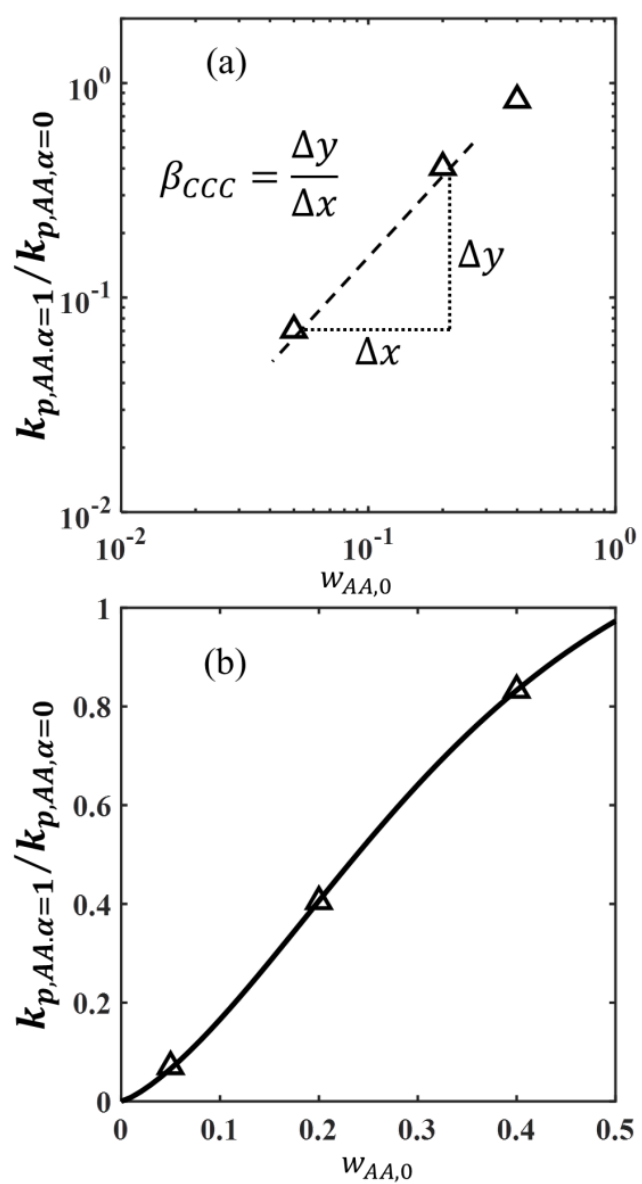

Fig. 5. Reduced propagation rate coefficient of fully ionized AA as a function of monomer concentration. (a) log-log plot of the experimental data (triangles) with the interpretation of the model parameter $\beta_{C C C}$; (b) comparison between experimental data (triangles) and simulated curves using Equation 32 (solid line).

It is also worth pointing out that the amount of experimental data taken into account to carry out the curve fitting is in this case relatively small compared with the number of fitting parameters in Equation 32. Nevertheless, these data have been determined by PLP-SEC, which is the best-known method to estimate propagation rate coefficients with high accuracy [35,36]. 
At this stage of the model development, we prioritize the consistency between the value of the reduced propagation rate coefficient of AA as a function of monomer concentration calculated through Equation 32 and the corresponding experimental estimation determined by PLP-SEC. Of course, the evaluation of the parameters in Equation 32 can be improved providing that additional information about the propagation kinetics of AA are made available.

Composition data. The remaining 8 parameters have been estimated by fitting the available experimental data of copolymer composition as a function of total monomer conversion. To this purpose, all of the experimental results obtained in this work at $\alpha=1$ and $f_{A m, 0}=0.5$ are considered (cf. Fig.s 1-4 and Table 1). In addition, experimental data of composition from Preusser et al. obtained at different degrees of ionization of AA as well as at different values of initial monomer composition are considered [19]. The estimated parameter values at $50^{\circ} \mathrm{C}$ are summarized in Table 4, whereas the comparison between experimental and simulated data is shown in Fig.s 6 and 7 as comonomer composition vs. conversion. The parameter optimization has been carried out by minimizing the square of the absolute discrepancy between the experimental and simulated composition versus conversion data using a genetic algorithm implemented in Matlab. 

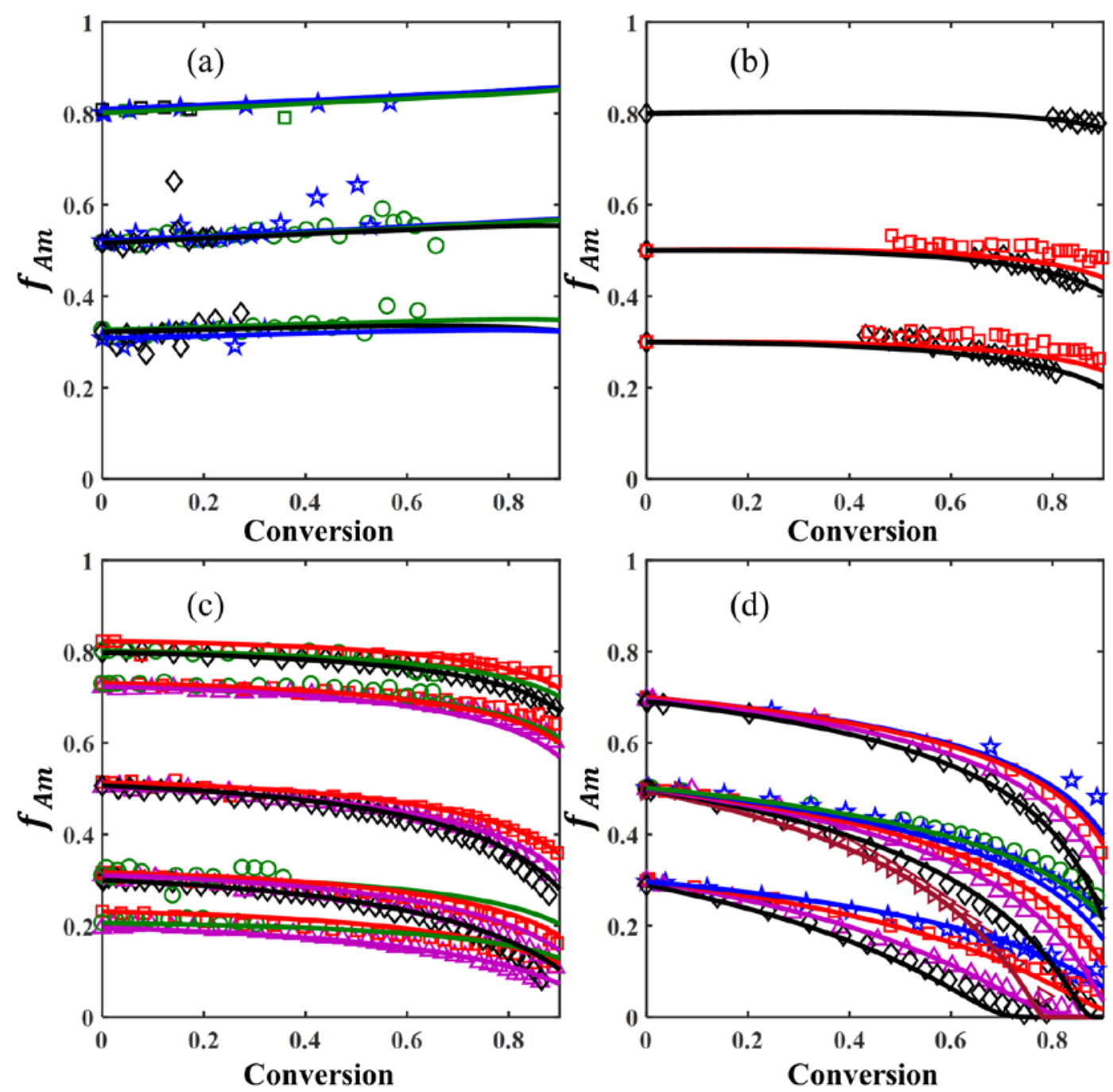

Fig. 6. Comparison between experimental (symbols) and simulated (solid lines) data of residual monomer mixture composition $f_{A m}$ as a function of conversion for the copolymerization of Am with $\mathrm{AA}$ at $50^{\circ} \mathrm{C}$ and initial monomer concentration equal to 1 (brown), 5 (black), 10 (violet), 20 (red), 30 (blue), and 40 wt\% (green). Initial degree of ionization (a) $\alpha=0.3$, (b) $\alpha=0.5$; (c) $\alpha=0.7$; (d) $\alpha=1$. The experimental data (with the exception of fully ionized AA at $f_{A m, 0}=0.5$ ) were taken from Preusser et al. [19]. 

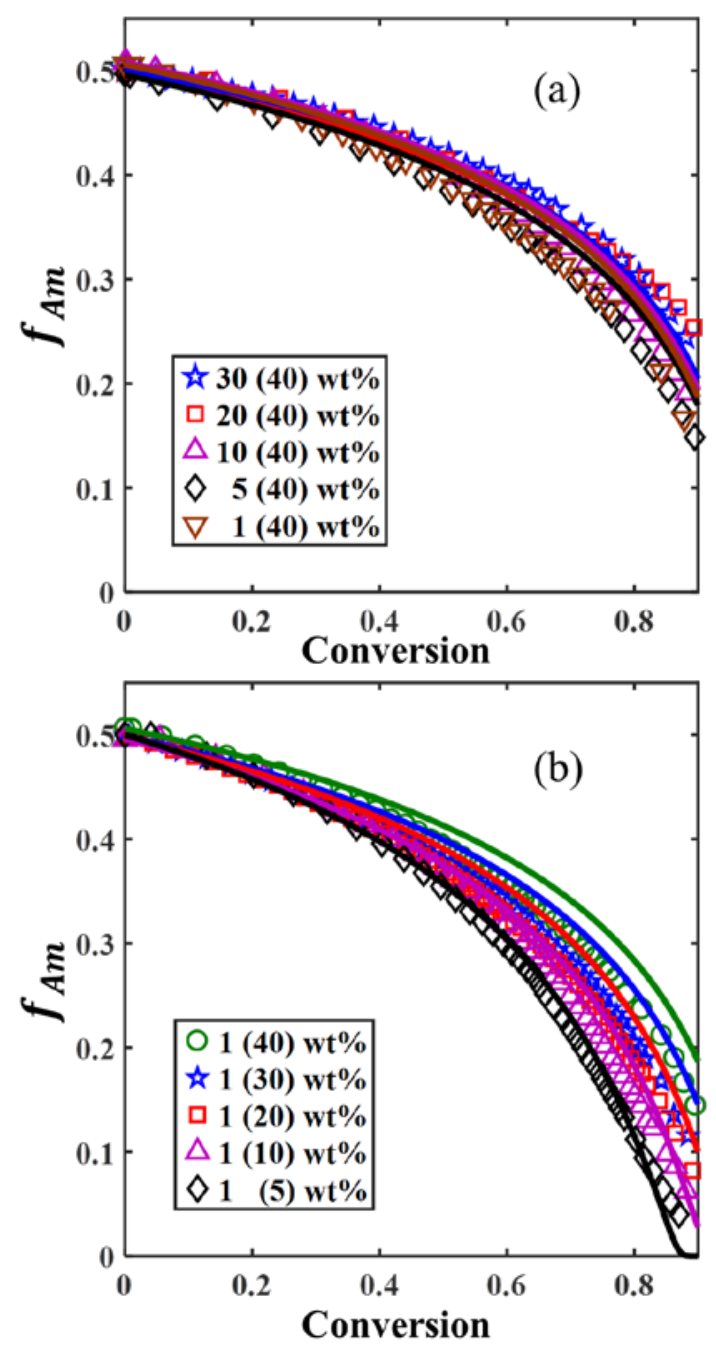

Fig. 7. Comparison between experimental (symbols) and simulated (solid lines) data of residual monomer mixture composition as a function of conversion for the copolymerization of Am with fully ionized AA at $50^{\circ} \mathrm{C}$ with salt addition. The initial weight fraction of total monomer is detailed in the legend, where the equivalent weight fraction of total monomer obtained after salt addition is reported within brackets. Reactions carried out at (a) constant ionic strength; (b) constant initial monomer concentration. 
Table 4. Optimized values of the model parameters estimated by fitting the equations to the experimental data of composition. The kinetic rates $k_{D, X C C}$ and $k_{D, C X C}$ are given in $\left(\mathrm{L} \mathrm{mol}^{-1} \mathrm{~s}^{-}\right.$ $\left.{ }^{1}\left(\mathrm{~kg} \mathrm{~mol}^{-1}\right)^{\beta}\right)$, whereas all other parameters are dimensionless.

\begin{tabular}{cc}
\hline Parameter & Optimized value \\
\hline$k_{D, X C C}$ & $6.96 \cdot 10^{5}$ \\
$\beta_{X C C}$ & 0.0198 \\
$k_{D, C X C}$ & $1.26 \cdot 10^{5}$ \\
$\beta_{C X C}$ & 2.58 \\
$r_{A m / A-}$ & 1.89 \\
$r_{A-/ A m}$ & 1.60 \\
$r_{H A / A-}$ & 1.90 \\
$r_{A-/ H A}$ & \\
\hline
\end{tabular}

The model is capable of reproducing nicely the effect of monomer concentration on the composition behavior in the experiments without salt addition, with some limited discrepancy at the highest monomer content (Fig. 6). The model performances are comparable with those of the empirical model developed by Preusser et al. to simulate the same set of experimental data [19]. About the experiments with salt addition, the model slightly overestimates the effect of copolymer enrichment in Am upon increasing the electrolyte concentration starting from the same monomer content especially at the largest electrolyte concentration, as shown in Fig. 7b. 
On the other hand, the simulation of the non-electrostatic effect due to increasing monomer concentration is not reproduced well, as shown in Fig. 7a. The only model feature accounting for a non-electrostatic effect due to increasing monomer concentration is the intrinsic dependence of the propagation rate coefficients of Am and HA on the monomer weight fraction $[33,34]$. This effect is well known for water-soluble monomers and has been explained in terms of stabilization of the transition state in the propagation step due to its interactions with the surrounding environment [33].

To conclude, looking at the global effect of the monomer concentration on the composition behavior of the system, the contribution of the electrostatic terms in the equations appears to partly compensate some non-electrostatic effect of the monomer concentration not yet adequately explained: accordingly, even though the overall fit of the experimental data in Fig. 6 remains satisfactory, the overestimation of the composition drift predicted by the model in Fig. 7 - which is uniquely due to electrostatic effects since the monomer concentration is constant - could be imputed to this model lack.

Model validation. The reliability of the model parameter values has been finally assessed by comparing the model predictions of reaction kinetics with the corresponding experimental data. Specifically, the propagation rate coefficient of MAA as a function of concentration and degree of ionization of the monomer has been measured by Lacik et al. in a wide range of temperatures [35]. The same rate coefficient can be predicted as a function of the degree of dissociation of AA and monomer concentration starting from the propagation rate coefficient of AA, taking advantage of the reduced propagation rate coefficients discussed before:

$$
k_{p, M A A}(\alpha)=k_{p, A A}(\alpha) \frac{k_{p, M A A . \alpha=0}}{k_{p, A A . \alpha=0}}
$$

where $k_{p, A A}(\alpha)$ is defined as in Equation 19, while $k_{p, A A . \alpha=0}$ (i.e., $k_{p, H A / H A}^{0}$ ) and $k_{p, M A A . \alpha=0}$ are taken from the literature [34,35]. A comparison between model predictions and experimental results at $50^{\circ} \mathrm{C}$ is shown in Fig. 8 . The model can reproduce very nicely the effects 
of monomer concentration and ionization on the propagation rate coefficient; it should be noted that for $\alpha=0$ the model equation for $k_{p, M A A}$ corresponds to the literature expression.

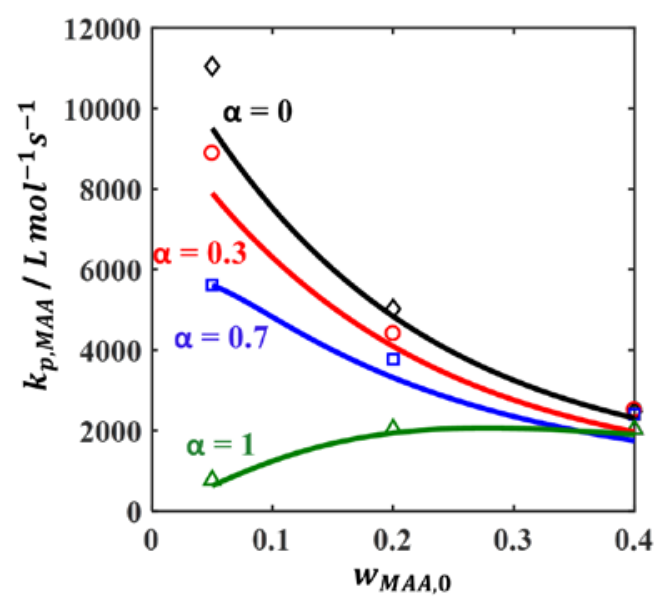

Fig. 8. Comparison between experimental (symbols) and simulated (solid lines) values of the propagation rate coefficient of MAA as a function of concentration and degree of ionization of the monomer at $50^{\circ} \mathrm{C}$. The experimental data are taken from Lacik et al. (table 2).[35]

This same model can be also used to evaluate the Mayo-Lewis plot for Am/AA through Equation 20 and to compare the result with the copolymerization diagram estimated by Rintoul et al. as a function of $\mathrm{pH}$ [17]. The predicted curves reported in Fig. 9 are overall in good agreement with the corresponding experimental data. In the case of fully ionized AA (i.e., pH $=12$ ) the model overestimates $F_{A m}$ when the Am fraction in the monomer mixture approaches zero. From Equations 20 and 22, the slope of the Mayo-Lewis plot in this condition can be expressed as follows:

$$
\left.\frac{d F_{A m}}{d f_{A m}}\right|_{f_{A m}=0}=\frac{1}{r_{A A}}
$$

In the limiting case of $f_{A m}=0$ and very high $\mathrm{pH}$ (i.e., AA is fully ionized and $\alpha_{M}=\alpha_{P}=1$ ), the reactivity ratio $r_{A A}$ defined by Equation 28 becomes:

$$
r_{A A}=r_{A-/ A m}\left(1+\frac{k_{p, H A / H A}^{0} S_{A-/ H A}}{k_{D, C C C} C_{E}^{\beta C C C}}\right)^{-1}
$$




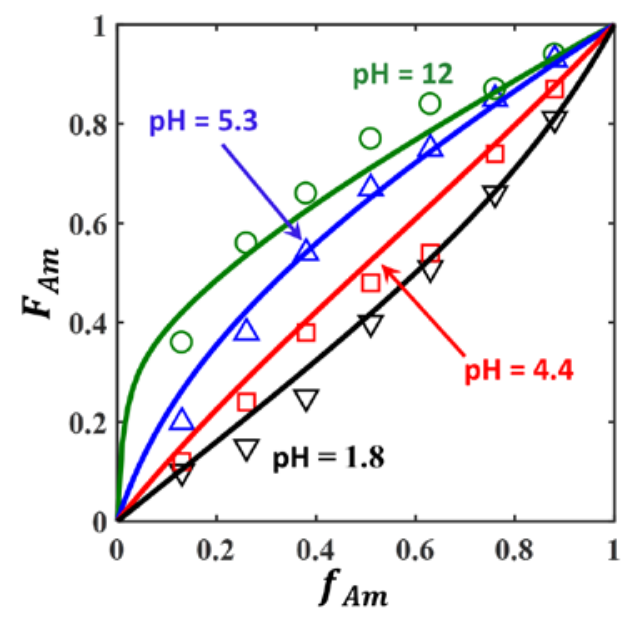

Fig. 9. Comparison between experimental (symbols) and simulated (solid lines) values of the Mayo-Lewis plot of Am/AA copolymerization as a function of the $\mathrm{pH}$ and at monomer concentration of $0.4 \mathrm{~mol} \mathrm{~L}^{-1}$. The experimental data were taken from Rintoul et al. (Fig. 4) [17].

Using the previously estimated model parameter values and a monomer concentration equal to $0.4 \mathrm{~mol} \mathrm{~L}^{-1}$ (as in the experiments by Rintoul et al.) to evaluate the electrolyte concentration, $r_{A A}=0.037$ is predicted. A much larger value (0.32) is reported by Rintoul et al. for the same parameter as determined by fitting of the composition data at $\mathrm{pH}=12$. This discrepancy is reflected by the larger initial slope of the simulated Mayo-Lewis curve at $\mathrm{pH}=12$ and $f_{A m}=0$ in Fig. 9 and the corresponding mismatch with the first experimental value at low Am fraction in the monomer mixture. It should be noted that the very small value of $r_{A A}$ calculated by Equation 37 is mainly determined by a large ratio $k_{p, H A / H A}^{0} s_{A-/ H A} / k_{D, C C C} C_{E}^{\beta C C C}$, thus it is primarily function of the electrostatic interaction parameters governing the propagation of nonionized AA. Nevertheless, these same parameter values enable the good prediction of PLP data of ionized AA propagation by Equation 31. 


\section{Conclusion}

The copolymerization of Am with AA in aqueous solution has been investigated by in-situ ${ }^{1} \mathrm{H}$ NMR with focus on copolymer composition. The experiments have been carried out at a 1:1 molar ratio between the two monomers, and employing AA in its fully ionized form ( $\alpha=$ 1) in order to deepen the understanding of the charge interactions on the reaction kinetics. The results showed that the incorporation of ionized AA in the copolymer can be enhanced either by increasing the total amount of monomer or by adding salt $(\mathrm{NaCl})$ to the reaction medium. This feature can be explained in terms of an effect of the solution ionic strength on the propagation reaction kinetics: the repulsion forces between the ionized AA repeat units in the active polymer chain and the approaching ionized AA monomer are reduced due to shielding of the charges by the addition of electrolytes to the solution. The reproduction of the monomer concentration effect by addition of salt revealed to be effective only at low monomer concentration (i.e., 1-5 wt\%): in such conditions, the propagation kinetics appears to be fully controlled by the electrostatic-driven diffusion limitations, and it is largely sensitive to changes in the ionic strength. On the other hand, at higher monomer concentration (i.e., 30-40 wt\%) the increase in ionic strength due to salt addition showed a much smaller, almost negligible impact onto the composition behavior compared to a mere increase in monomer concentration, suggesting that the electrostatic interactions play a minor role. In this case, the reaction is mainly controlled by the intrinsic kinetics of propagation, and the effect of monomer concentration can be ascribed to a different degree of stabilization of the transition state according to the composition of its surrounding environment.

In the attempt to elucidate the competition between electrostatic and non-electrostatic effects on the composition behavior due to an increase in monomer concentration, a mathematical model has been developed, which calculates the copolymer composition as a function of conversion. The dependence of the reactivity ratios on the electrostatic interactions has been 
expressed in accordance with the DLVO theory, based on the rate law of propagation of ionized monomers proposed in a previous work [13]: namely, a diffusion-dependent contribution function of the ionic strength is introduced in addition to the intrinsic kinetics term to evaluate $k_{p}$. The corresponding kinetic model of copolymerization involving a permanently-charged monomer has been extended in this work to cover any ionization degree of AA. As a further improvement to the previous model, the penultimate unit effect for the electrostatic interactions has been considered, according to the relatively small distance between the approaching charges in the transition state of AA propagation. The model parameters have been estimated by fitting the equations to a large set of experimental data, including own data about fully ionized AA with salt addition as well as literature data obtained at various degrees of ionization of AA. The developed model is capable to reproduce nicely the experimental data in a wide range of reaction conditions. Moreover, the reliability of the parameter values was confirmed by comparison of the model predictions with published composition data not used for the parameter estimation. Remarkably, the model equations and parameter values defining the propagation rate of AA as a function of its degree of ionization and concentration showed to be consistent with PLP estimations.

The presented approach can be extended to other systems involving water-soluble monomers, particularly in presence of ionizable groups. However, given the large number of model parameters, their reliable estimation is not trivial, and the use of combined experimental information (copolymer composition by in-situ NMR, propagation rate coefficients from independent PLP data, molecular weight distribution by SEC) should always be considered. In particular, the electrostatic interaction parameters introduced by equations 7-11 are expected to be weakly dependent on the specific monomer pair under consideration, thus their estimated values could be applied to model the kinetic behavior of different monomers sharing similar charge-bearing groups. 


\section{SUPPLEMENTARY MATERIAL}

Fig. S1. ${ }^{1} \mathrm{H}$ NMR spectrum of the copolymerization of Am with AA at $50^{\circ} \mathrm{C}$. Fig. S2. Experimental results of residual monomer mixture composition as a function of conversion for the copolymerization of Am with fully ionized $\mathrm{AA}$ at $50^{\circ} \mathrm{C}$ and comparison with the results obtained at Queen's. Fig. S3. Optimized structures of oligomers of ionized AA and DMAEAQ with the distances between the side-chain charges. Calculation of the degrees of dissociation of AA monomer and AA polymer units as a function of $\mathrm{pH}$. Definition of the pseudo-binary rate coefficients.

\section{FUNDING}

This work was supported by the Swiss National Science Foundation [grant number 200021.153403/1].

CONFLICTS OF INTEREST

Conflicts of interest: none.

\section{ACKNOWLEDGMENT}

The financial support from the Swiss National Science Foundation with Grant Number 200021.153403/1 is gratefully acknowledged.

\section{REFERENCES}

[1] V.A. Myagchenkov, V.F. Kurenkov, Applications of Acrylamide Polymers and Copolymers: A Review, Polym. Plast. Technol. Eng. 30 (1991) 109-135. 
[2] D. Hunkeler, J. Hernandez-Barajas, Polyacrylamides, Handbook of Thermoplastics, Marcel Dekker, New York, 1997.

[3] K.C. Taylor, H.A. Nasr-El-Din, Water-Soluble Hydrophobically Associating Polymers for Improved Oil Recovery: a Literature Review, J. Pet. Sci. Eng. 19 (1998) 265-280.

[4] F.L. Buchholz, Polyacrylamides and Poly(acrylic acids), Ullmann's Encyclopedia of Industrial Chemistry, Wiley-VCH Verlag GmbH \& Co. KGaA, 2000.

[5] A.V. Dobrynin, M. Rubinstein, Theory of Polyelectrolytes in Solutions and at Surfaces, Prog. Polym. Sci. 30 (2005) 1049-1118.

[6] S.K. Tripathy, J. Kumar, H.S. Nalwa, Handbook of Polyelectrolytes and Their Applications, Eds., ASP, Stevenson Ranch, CA, 2002.

[7] F. Oosawa, Polyelectrolytes, Marcel Dekker, New York, 1971.

[8] A. Paril, A.M. Alb, A.T. Giz, H. Catalgil-Giz, Effect of Medium pH on the Reactivity Ratios in Acrylamide Acrylic Acid Copolymerization, J. Appl. Polym. Sci. 103 (2007) 968974.

[9] G.S. Manning, Limiting Laws and Cunterion Condensation in Polyelectrolyte Solutions I. Colligative Properties, J. Chem. Phys. 51 (1969) 924-933.

[10] M. Riahinezhad, N. Kazemi, N. McManus, A. Penlidis, Effect of Ionic Strength on the Reactivity Ratios of Acrylamide/Acrylic Acid (sodium acrylate) Copolymerization, J. Appl. Polym. Sci. 131 (2014) 40949.

[11] R. Losada, C. Wandrey, Non-Ideal Polymerization Kinetics of a Cationic Double Charged Acryl Monomer and Solution Behavior of the Resulting Polyelectrolytes, Macromol. Rapid Commun. 29 (2008) 252-257.

[12] V.F. Kurenkov, I.N. Nadezhdin, O.A. Antonovich, F.I. Lobanov, Phase Separation in Aqueous Solutions of Binary Copolymers of Acrylamide with Sodium 2-Acrylamido-2Methylpropanesulfonate and Sodium Acrylate, Russ. J. Appl. Chem. 29 (2004) 804-808.

[13] D. Cuccato, G. Storti, M. Morbidelli, Experimental and Modeling Study of Acrylamide Copolymerization with Quaternary Ammonium Salt in Aqueous Solution, Macromolecules 48 (2015) 5076-5087.

[14] A. Chapiro, Polymerization and Copolymerization in Associated Monomer Aggregates, Eur. Polym. J. 9 (1973) 417-427.

[15] W.R. Cabaness, T.Y. Lin, C. Párkányi, Effect of $\mathrm{pH}$ on the Reactivity Ratios in the Copolymerization of Acrylic Acid and Acrylamide, J. Polym. Sci., Part A-1: Polym. Chem. 9 (1971) 2155-2170.

[16] S. Ponratnam, S.L. Kapur, Reactivity Ratios of Ionizing Monomers in Aqueous Solution: Copolymerization of Acrylic and Methacrylic Acids with Acrylamide, Makromol. Chem. 178 (1977) 1029-1038.

[17] I. Rintoul, C. Wandrey, Polymerization of Ionic Monomers in Polar Solvents: Kinetics and Mechanism of the Free Radical Copolymerization of Acrylamide/Acrylic Acid, Polymer 46 (2005) 4525-4532.

[18] M. Riahinezhad, N. Kazemi, N. McManus, A. Penlidis, Optimal Estimation of Reactivity Ratios for Acrylamide/Acrylic Acid Copolymerization, J. Polym. Sci., Part A: Polym. Chem. 51 (2013) 4819-4827.

[19] C. Preusser, I.H. Ezenwajiaku, R.A. Hutchinson, The Combined Influence of Monomer Concentration and Ionization on Acrylamide/Acrylic Acid Composition in Aqueous Solution Radical Batch Copolymerization, Macromolecules 49 (2016) 4746-4756.

[20] A. Paril, A. Giz, H. Catalgil-Giz, Composition Control Through pH and Ionic Strength During Acrylic Acid/Acrylamide Copolymerization, J. Appl. Polym. Sci. 127 (2013) 35303536. 
[21] M. Riahinezhad, N. McManus, A. Penlidis, Effect of Monomer Concentration and pH on Reaction Kinetics and Copolymer Microstructure of Acrylamide/Acrylic Acid Copolymer, Macromol. React. Eng. 9 (2015) 100-113.

[22] C. Preusser, R.A. Hutchinson, An In-Situ NMR Study of Radical Copolymerization Kinetics of Acrylamide and Non-Ionized Acrylic Acid in Aqueous Solution, Macromol. Symp. 333 (2013) 122-137.

[23] A.P. Haehnel, M. Stach, A. Chovancova, J.M. Rueb, G. Delaittre, A.M. Misske, I. Lacik, C. Barner-Kowollik, (Meth)acrylic Monomers with Heteroatom-Containing Ester Side Chains: a Systematic PLP-SEC and Polymerization Study, Polym. Chem. 5 (2014) 862-873.

[24] U.C. Palmiero, A. Chovancova, D. Cuccato, G. Storti, I. Lacík, D. Moscatelli, The RAFT Copolymerization of Acrylic Acid and Acrylamide, Polymer 98 (2016) 156-164.

[25] A.D. Becke, Density-Functional Thermochemistry .3. The Role of Exact Exchange, J. Chem Phys. 98 (1993) 5648-5652.

[26] W.Y.C. Lee, R.G. Parr, Development of the Colle-Salvetti Correlation-Energy Formula into a Functional of the Electron-Density, Phys. Rev. B 37 (1988) 785-789.

[27] R. Ditchfield, W.J. Hehre, J.A. Pople, Self-Consistent Molecular-Orbital Methods .9. Extended Gaussian-Type Basis for Molecular-Orbital Studies of Organic Molecules, J. Chem. Phys. 54 (1971) 724-728.

[28] S. Miertus, E. Scrocco, J. Tomasi, Electrostatic interactions of a solute with a continuum. A direct utilization of ab initio molecular potentials for the prevision of solvent effects, Chem. Phys. 55 (1981) 117-129.

[29] H. Kattner, M. Buback, EPR Study of Backbiting in the Aqueous-Solution Polymerization of Acrylamide, Macromol. Rapid Commun. 36 (2015) 2186-2191.

[30] J. Barth, M. Buback, Termination and Transfer Kinetics of Sodium Acrylate Polymerization in Aqueous Solution, Macromolecules 45 (2012) 4152-4157.

[31] R. Arnold, The Titration of Polymeric Acids, J. Colloid Sci. 12 (1957) 549-556.

[32] I. Skeist, Copolymerization: the Composition Distribution Curve, J. Am. Chem. Soc. 68 (1946) 1781-1784.

[33] I. Lacík, A. Chovancova, L. Uhelska, C. Preusser, R.A. Hutchinson, M. Buback, PLPSEC Studies into the Propagation Rate Coefficient of Acrylamide Radical Polymerization in Aqueous Solution, Macromolecules 49 (2016) 3244-3253.

[34] N.F.G. Wittenberg, C. Preusser, H. Kattner, M. Stach, I. Lacik, R.A. Hutchinson, M. Buback, Modeling Acrylic Acid Radical Polymerization in Aqueous Solution, Macromol. React. Eng. 10 (2016) 95-107.

[35] I. Lacik, L. Ucnova, S. Kukuckova, M. Buback, P. Hesse, S. Beuermann, Propagation Rate Coefficient of Free-Radical Polymerization of Partially and Fully Ionized Methacrylic Acid in Aqueous Solution, Macromolecules 42 (2009) 7753-7761.

[36] K.B. Kockler, A.P. Haehnel, T. Junkers, C. Barner-Kowollik, Determining Free-Radical Propagation Rate Coefficients with High-Frequency Lasers: Current Status and Future Perspectives, Macromol. Rapid Commun. 37 (2016) 123-134. 